\title{
A Posted-Price Auction for Heterogeneous Spectrum Sharing under Budget Constraints
}

\author{
Wen-Hsiang Lai \\ Department of Communication \\ Engineering \\ National Central University \\ Taoyuan, Taiwan
}

\author{
Pavol Polacek \\ Department of Communication \\ Engineering \\ National Central University \\ Taoyuan, Taiwan
}

\author{
Chih-Wei Huang \\ Department of Communication \\ Engineering \\ National Central University \\ Taoyuan, Taiwan
}

\begin{abstract}
The concept of cognitive radio is emerging as a platform for designing next generation wireless networks with dynamic resource sharing among machines. However, how to efficiently and fairly share the spare channels is still a topic under intensive research. This paper investigates a spectrum trading problem under relatively realistic settings, where heterogeneous channels under buyers' budget constraints are specifically considered, while maintaining incentive compatibility and individual rationality. The proposed auction game consists of a price-setting primary owner (PO), targeting on maximizing its total revenue, and secondary users (SUs), bidding channels for reasonable values. To handle channel heterogeneity, SUs provide independent valuation according to observed quality on different channels. The PO adopts a sequential arbitrary-order mechanism for flexible deployment and achieving aforementioned economic properties in polynomial time. Finally, the numerical results show performance improvements in PO revenue and SU utility over reference approaches.
\end{abstract}

\section{Categories and Subject Descriptors}

G.1.6 [Mathematics of Computing]: Optimization-Linear programming; C.2.1 [Computer-communication Networks]: Network Architecture and Design-Wireless communications

\section{Keywords}

Spectrum sharing, cognitive radio, game theory, auction algorithm.

\section{INTRODUCTION}

The concept of cognitive radio (CR) is emerging as a new paradigm for designing next generation wireless networks with dynamic spectrum sharing. In contrast to traditional spectrum sharing approaches, CR users need to be aware of the ambient radio condition and adjust the transmission parameters based on interactions with environment and other users. Frequency agile secondary (unlicensed) users (SU) share frequency spectrum with primary (licensed) owners (POs) to improve channel usage and overall available bandwidth [4].

Game theory is one of key mathematical tools to model and analyze the spectrum trading process under the interactive nature of CR scenarios. Among various trading mechanisms, auction games have been considered as efficient and important means of spectrum allocation [9].

The impact of heterogeneity on spectrum sharing has been studied to some extent. Ma et al. [6] claimed to provide the first attempt to model channel heterogeneity into CR spectrum sharing. In [5], the study consider a more general and more realistic case where channels have different qualities. Also, they allow SUs to express their preferences for each channel separately. They design an auction-based spectrum sharing mechanism where the SUs bid to buy spectrum bands from the PO, who acts as the auctioneer, selling idle spectrum bands to make a profit. In [10], they perform subchannel allocation to roughly satisfy heterogeneous users' rate requirements and remove the intractable integer constraints of the optimization problem. The related works are either over simplifying the effect of wireless channel quality or assuming bidders to have unlimited budgets.

Therefore, we argue that both channel heterogeneity and SUs' budget should be jointly considered. We also know that straightforward Vickrey-Clarke-Groves approach can not effectively deal with budget constraints [1]. In this work, we investigate a realistic spectrum trading problem considering heterogeneous channels under buyers' budget constraints, while maintaining incentive compatibility and individual rationality. The proposed auction game consists of a price-setting PO targeting on maximizing its total revenue and SUs bidding channels for reasonable values. To handle channel heterogeneity, SUs provide independent valuation according to observed quality on different channels. To accommodate SUs randomly requesting for transmission in machine-to-machine scenarios, the PO adopts a sequential arbitrary-order mechanism to achieve aforementioned economic properties and allocate the spectrum in polynomial time. Finally, the numerical results show performance improvements in SU utility and PO revenue over homogeneous sharing and maximum weight matching approaches. 


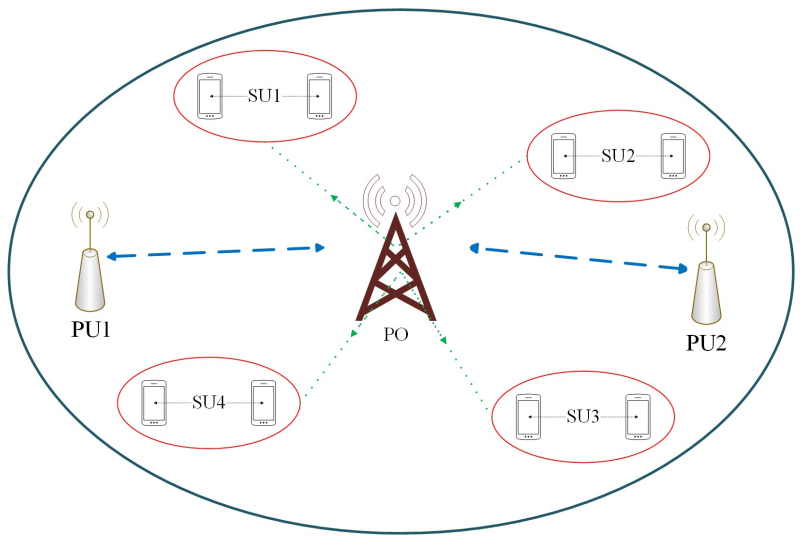

Figure 1: A cognitive radio network with four SUs operating over an area covered by a licensed PO. The spectrum under-used by primary users (PUs) are shared with SUs.

The rest of this paper is organized as follows: Section 2 introduces the system model of spectrum sharing. Section 3 formulates the heterogeneous auction problem. The results of spectrum sharing simulations are presented in Section 4 . Final conclusions are drawn in Section 5.

\section{SYSTEM MODEL}

\subsection{Spectrum Trading in CR Networks}

We consider a CR network with a set $\mathcal{S}$ of $S$ SUs operating over an area covered by a licensed PO as illustrated in Figure 1. The PO is able to share under-used spectrum to SU $s$ at price $p_{s}$. The SU refers to a pair of secondary transmitter and receiver equipped with spectrum sensing capability. Overall, the PO is interested in selling spare channels for extra revenue, while SUs are interested in buying resources to gain service utility.

We adopt auction-based mechanisms treating PO as auctioneer, SUs as bidders, and unused channels as auction items. During the deal making process, SUs propose the channel valuation and desired quantity to $\mathrm{PO}$, and then $\mathrm{PO}$ determines the actual channel assignment and the price each SU has to pay. We assume all players are rational and conform to the rules described above.

\subsection{Utility and Revenue under Heterogeneous Channels}

We assume heterogeneous wireless channels in our system. SU pairs experience different signal quality across a set $\mathcal{N}$ of $N$ available channels resulting in distinct potential utility gain. The channel preference, or valuation $v$, is defined as the benefit of obtaining a channel by an SU. Since more data transfer capacity generally provides better quality for overlaying services, SUs' valuation for a channel is directly related to the spectrum efficiency $[2]$ in bit $/ \mathrm{s} / \mathrm{Hz}$. Based on the commonly used adaptive modulation concept, the spectral efficiency of $\mathrm{SU} s$ on channel $n$ is:

$$
q_{s n}=\log _{2}\left(1+\frac{1.5}{\ln \left(0.2 / B E R_{s}\right)} \overline{S N R}_{s n}\right)
$$

where $B E R_{s}$ is the target bit-error rate of SU $s$, and $\overline{S N R}_{s n}$ is the average received signal-to-noise ratio ( $\mathrm{SNR}$ ) of $\mathrm{SU} s$ on channel $n$. The valuation is $v_{s n}=\alpha \cdot q_{s n}$ and $\alpha$ is a coefficient. The actual valuations are private information of SUs and not known by PO or other SUs. Therefore, the PO relies on incentive compatible auction mechanisms, to make sure that SUs have a motive to reveal their true valuations.

For channel assignment, we assume that each channel can only be used by one SU at a time, but each SU can simultaneously use more than one channels. The PO can not assign more channels to an $\mathrm{SU}$ than its demand $D_{s}$, while an SU can only pay for channels within its preset budget $B_{s}$. The utility $u_{s}$ of SU, $s$, is the difference between valuations for the obtained channels and the total price to pay:

$$
u_{s}=\sum_{n \in \mathcal{N}} v_{s n}-p_{s}
$$

The goal of the PO is to maximize its revenue. The PO has incentive to attract more SUs with a fair and individually profitable game, so the total revenue $R=\sum_{s \in \mathcal{S}} p_{s}$, can be maximized. Another important goal of the auction is social welfare, defined as PO profit plus the sum of the SUs' utilities. Social welfare $S W$ is [5]:

$$
S W=R+\sum_{s \in \mathcal{S}} u_{s}
$$

\section{BUDGET CONSTRAINED HETEROGE- NEOUS CHANNEL SHARING}

In this section, we formulate a realistic heterogeneous channel sharing problem with incentive compatibility and individual rationality in mind. Specially, each SU pair can set a budget constraint to reflect its potential gain from the running application service. After proper linear programming formulation, we feed polynomial-time-solvable parameters into a posted-price auction mechanism for resource allocation.

\subsection{The SU Channel Valuation}

For all SUs and channels, we round valuations $v_{s n}$ to integers in the range $\left[1, \ldots, L_{s n}\right]$ and fit into a probability mass function $f_{s n}(\cdot)$. As a preference for lower price leads to higher utility, we adjust the probability distribution using the Marshall-Olkin discrete uniform distribution [8]. Adding the parameter $k$, we get:

$$
f^{\prime}\left(v, L_{s n}, k\right)=\frac{L_{s n} \cdot k}{\left[L_{s n} \cdot k+(1-k) v\right]\left[L_{s n} \cdot k+(1-k)(v-1)\right]}
$$

where $0<k<1$ and $v=1,2, \ldots, L_{s n}$. As a result, lower bids will be drawn with a higher probability.

We further define $\mathcal{V}_{s n}=\min \left(v_{s n}, \frac{B_{s}}{4}\right)$ and its range is $\left[1, \ldots, R_{s n}\right]$. The probability mass function $g_{s n}(r)$ can be defined as:

$g_{s n}(r)=\operatorname{Pr}\left[\mathcal{V}_{s n}=r\right]= \begin{cases}f_{s n}^{\prime}\left(r, R_{s n}, k\right), & \text { if } r<B_{s} / 4 \\ \sum_{v=r}^{L_{s n}} f_{s n}^{\prime}\left(r, R_{s n}, k\right), & \text { if } r=B_{s} / 4 \\ 0, & \text { otherwise. }\end{cases}$

In order to maximize the PO's profit under bidders' preferences, we use the virtual valuation $\hat{v}$ [7]. The function is 
defined for all $r \in R_{s n}$ as:

$$
\hat{v}_{s n}(r)=r-\frac{\operatorname{Pr}\left[\mathcal{V}_{s n}>r\right]}{\operatorname{Pr}\left[\mathcal{V}_{s n}=r\right]}
$$

where the distribution of $\hat{v}_{s n}(r)$ qualifies as a monotonous hazard rate [1].

\subsection{The Channel Auction Problem}

For the channel allocation, we formulate a linear program to devise the sequential posted price auction leading to the proposed algorithm.

The maximization of $\mathrm{PO}$ revenue, while respecting the budgetary and demand constraints of the SUs, can be formulated as:

$$
\begin{array}{ll}
\text { Maximize } & \sum_{s \in \mathcal{S}} \sum_{n \in \mathcal{N}} \sum_{r \in R_{s n}} g_{s n}(r) \cdot \hat{v}_{s j}(r) \cdot z_{s n}(r) \\
\sum_{n \in \mathcal{N}} \sum_{r \in R_{s n}} g_{s n}(r) \cdot z_{s n}(r) \leq D_{s} & \forall s \in \mathcal{S} \\
\sum_{n \in \mathcal{N}} \sum_{r \in R_{s n}} g_{s n}(r) \cdot \hat{v}_{s j}(r) \cdot z_{s n}(r) \leq B_{s} & \forall s \in \mathcal{S} \\
\sum_{s \in \mathcal{S}} \sum_{r \in R_{s n}} g_{s n}(r) \cdot z_{s n}(r) \leq 1 & \forall n \in \mathcal{N} \\
z_{s n}(r) \in[0,1] & \forall s, n, r
\end{array}
$$

the variable $z_{s n}$ is the probability a channel $n$ is allocated to user $s$. The first constraints limits the per SU channel allocation to a predefined demand $D_{s}$. The second constraint means that the value of allocated channels can not exceed the budget $B_{s}$. The third constraint allows each channel to be allocated only once. The last constraint limits the range of $z_{s n}$.

Due to the use of virtual valuation, the above linear program can be solved in polynomial time satisfying incentive compatibility and individual rationality. Therefore, an auction algorithm can be develop based on the formulated problem.

\subsection{The Sequential Arbitrary-Order Mecha- nism}

The mechanism is incentive compatible and individually rational with following features:

1. Maximizing non-negative SUs utility.

2. Truth telling bidders leading to predictable auction results.

3. Maximizing the number of used channels, i.e. channel utilization.

We propose Sequential Arbitrary-order Mechanism (SAM) that has the desired economic properties, and leads to higher channel utilization and SU utility. The pseudocode of the SAM algorithm is in Algorithm 1.

Solve the linear program (7) and denote the solution as $z_{s n}^{*}$. The solution of the linear program enables us to select the potential payment $\tilde{r}_{s n}$ for all $(s, n)$, by decomposing $z_{s n}^{*}$ into components of a convex combination. Next, let $\left\{Y_{s n}\right\}$ be a set of $N \times S$ variables, such that $\operatorname{Pr}\left[Y_{s n}=1\right]=1 / 4$ and $\operatorname{Pr}\left[Y_{s n}=0\right]$ otherwise. Initialize the set $A$ to be the set of all available channels.

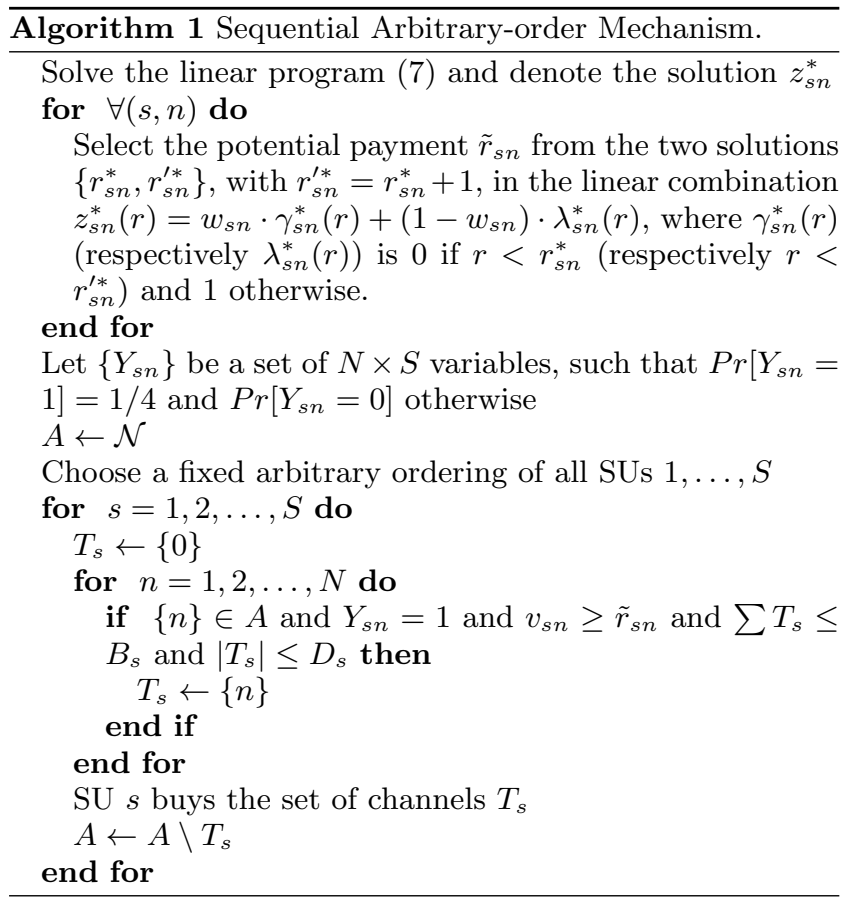

As the supply, demand, and budget constraints hold only in expectation, the pairs $(s, n)$ are not entirely independent, so obtaining a practical allocation is difficult. To get around these limitations, we visit the bidders in a arbitrary predetermined order and offer items with a probability of $1 / 4$ at a price $\tilde{r}_{s n}$. The SUs then use a knapsack heuristic approach to buy more and more channels in the set $T_{s}$, until the budget is used up or the demand is satisfied. SU $s$ buys the set of channels $T_{s}$ and these channels are not available to other SUs.

\section{SIMULATION RESULTS}

In this section, we compare the proposed SAM method with heterogeneous (SAMHe) and homogeneous (SAMHo) channel valuation, and maximum weight matching (MWM) done by Khaledi and Abouzeid [5].

The simulation was performed in Matlab with SNR value from -5 to $30 \mathrm{~dB}$, the number of SUs from 1 to 25 , and the number of available channels $N$ is 8 . The budget $B_{s}$ is randomly selected to be between $50 \%$ and $60 \%$ of the sum of valuation of SU $s$ of all channels. In the heterogeneous scenarios, each SU pair's valuation depends on the channel capacity between the two, while valuations are identical for all links in homogeneous cases. The linear program has been solved with convex programming solver [3]. We focus on competitive conditions characterizing low channel to SU ratios.

The MWM allocation process is based on bipartite graph for SUs and their valuation, after which the Hungarian algorithm is used to determine the channel allocation. The price an $\mathrm{SU}$ has to pay is equal to the externality caused. The $\mathrm{SU}$ budget is not considered during the original process. A new rule is added to prevent SUs from using channels exceeding its budgets.

Figure 2 shows the achieved average utility per SU. For all schemes, the utility decreases with higher SU numbers. For 


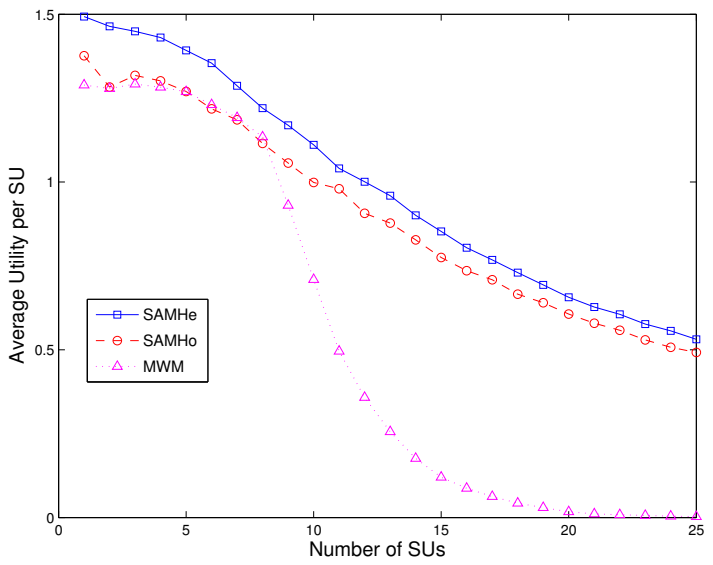

Figure 2: Average utility per SU. SAMHe and SAMHe both show a steady decrease in average utility. MWM however drops very sharply from the initial values after $8 \mathrm{SUs}$.

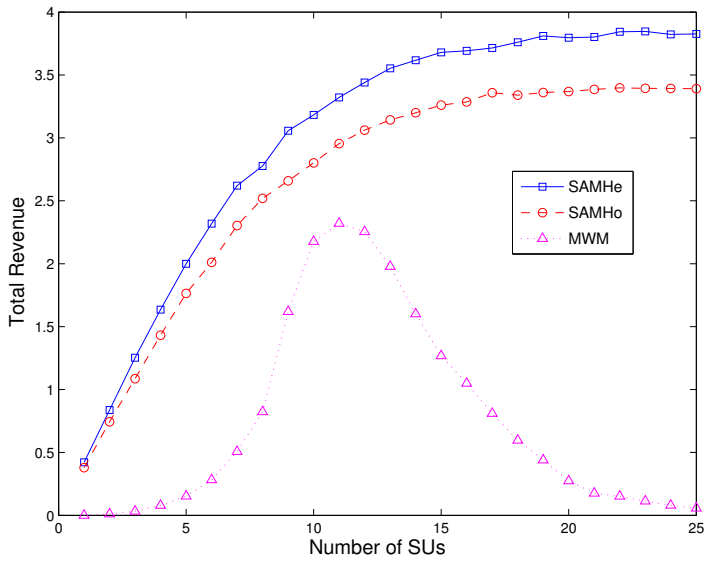

Figure 3: Total revenue obtained by the PO. Both SAM methods are able to increase the revenue until flattening from 15 SUs onwards. MWM starts at a low value, achieves its maximum at $11 \mathrm{SUs}$ and then starts dropping for higher SU numbers.

SAMHe and SAMHo, this decrease is steady, with SAMHe achieving overall higher utility. MWM starts at similar values as the former two schemes, but quickly drops after 8 SUs, due to the allocation exceeding its budgets.

In Figure 3, both SAM mechanisms can have stable profit when the number of SUs increases. The SAMHe achieves overall higher profits, when compared to SAMHo. The MWM method suffers from missing budget constrains during the allocation process. After achieving its maximum at $11 \mathrm{SUs}$, MWM quickly starts dropping along with increasing number of SUs.

With a limited number of channels and an ever increasing number of SUs, we see the average SU utility and total revenue changing. For the SAM schemes, the the utility slowly decreases and the revenue rises only to flatten, as the competition for channels increases, the utility value obtainable from the available channels is limited. For the MWM method, due to the increased competition, ever higher value channels are being allocated to SUs. In the next step however, it is checked whether the payment for these high value channels is within the SU budget. As this is often not the case, the rate of SUs, which have to reject their allocation increases, leading to the sudden drop from the maximum in Figure 2 and Figure 3. As for the rate of allocated channels, SAM schemes are able to continuously increase it until saturation and then keep it high, while the MWM scheme channel allocation rate drops after saturation, similarly to the drop in SU utility and system revenue.

\section{CONCLUSIONS}

This paper propose a sequential arbitrary-order spectrum sharing mechanism suitable for flexible deployment. The auction game can jointly handle wireless channel heterogeneity and secondary users' budget constraints. In competitive situation with low channel to SU ratio, the proposed auction scheme outperforms approaches based on homogeneous valuation or graph matching.

\section{Acknowledgment}

The research is based on work supported by the Ministry of Science and Technology (MOST) of Taiwan, under grant number MOST 104-2923-E-002-005-MY3.

\section{REFERENCES}

[1] S. Bhattacharya, G. Goel, G. Sreenivas, and K. Munagala. Budget-constrained auctions with heterogeneous items. Theory of computing, 8:429-460, 2012.

[2] A. Goldsmith. Variable-rate variable-power MQAM for fading channels. IEEE Transactions on Communications, 45(10):1218-1230, 1997.

[3] M. Grant and S. Boyd. CVX: Matlab Software for Disciplined Convex Programming, version 2.0 beta. \url\{http://cvxr.com/cvx\}, 2013.

[4] S. Haykin. Cognitive radio: brain-empowered wireless communications. IEEE Journal on Selected Areas in Communications, 23(2):201-220, Feb. 2005.

[5] M. Khaledi and A. Abouzeid. Auction-Based Spectrum Sharing in Cognitive Radio Networks with Heterogeneous Channels. In Information Theory and Applications Workshop, San Diego, USA, Feb. 2013.

[6] M. Ma and D. H. K. Tsang. Impact of Channel Heterogeneity on Spectrum Sharing in Cognitive Radio Networks. In IEEE International Conference on Communications, pages 2377-2382, 2008.

[7] R. B. Myerson. Optimal auction design. Mathematics of operations research, 6(1):58-73, 1981.

[8] E. Sandhya and C. Prasanth. Marshall-olkin discrete uniform distribution. Journal of Probability, 2014, 2014.

[9] B. Wang, Y. Wu, and K. R. Liu. Game theory for cognitive radio networks: An overview. Computer Networks, 54(14):2537-2561, Oct. 2010.

[10] S. Wang, Z.-H. Zhou, M. Ge, and C. Wang. Resource Allocation for Heterogeneous Cognitive Radio Networks with Imperfect Spectrum Sensing. IEEE Journal on Selected Areas in Communications, 31(3):464-475, Mar. 2013. 